\title{
Ciliopathy proteins regulate paracrine signaling by modulating proteasomal degradation of mediators
}

\author{
Yangfan P. Liu, ${ }^{1}$ I-Chun Tsai, ${ }^{1}$ Manuela Morleo, ${ }^{2}$ Edwin C. Oh, ${ }^{1}$ Carmen C. Leitch, ${ }^{3}$ \\ Filomena Massa, ${ }^{2}$ Byung-Hoon Lee, ${ }^{4}$ David S. Parker, ${ }^{1}$ Daniel Finley, ${ }^{4}$ \\ Norann A. Zaghloul,3 Brunella Franco,2,5 and Nicholas Katsanis'
${ }^{1}$ Center for Human Disease Modeling, Duke University, Durham, North Carolina, USA. ${ }^{2}$ Telethon Institute of Genetics and Medicine, Naples, Italy. ${ }^{3}$ Department of Medicine, Division of Endocrinology, Diabetes, and Nutrition, University of Maryland School of Medicine, Baltimore, Maryland, USA. ${ }^{4}$ Department of Cell Biology, Harvard Medical School, Boston, Massachusetts, USA. ${ }^{5}$ Department of Medical Translational Sciences, Federico II University, Naples, Italy.

\begin{abstract}
Cilia are critical mediators of paracrine signaling; however, it is unknown whether proteins that contribute to ciliopathies converge on multiple paracrine pathways through a common mechanism. Here, we show that loss of cilopathy-associated proteins Bardet-Biedl syndrome 4 (BBS4) or oral-facial-digital syndrome 1 (OFD1) results in the accumulation of signaling mediators normally targeted for proteasomal degradation. In WT cells, several BBS proteins and OFD1 interacted with proteasomal subunits, and loss of either BBS4 or OFD1 led to depletion of multiple subunits from the centrosomal proteasome. Furthermore, overexpression of proteasomal regulatory components or treatment with proteasomal activators sulforaphane (SFN) and mevalonolactone (MVA) ameliorated signaling defects in cells lacking BBS1, BBS4, and OFD1, in morphant zebrafish embryos, and in induced neurons from $O f d 1$-deficient mice. Finally, we tested the hypothesis that other proteasome-dependent pathways not known to be associated with ciliopathies are defective in the absence of ciliopathy proteins. We found that loss of BBS1, BBS4, or OFD1 led to decreased NF- $\kappa$ B activity and concomitant $I \kappa B \beta$ accumulation and that these defects were ameliorated with SFN treatment. Taken together, our data indicate that basal body proteasomal regulation governs paracrine signaling pathways and suggest that augmenting proteasomal function might benefit ciliopathy patients.
\end{abstract}

\section{Introduction}

In vertebrates, the cilium and the basal body are key components of paracrine signaling transduction. This has, in turn, suggested that phenotypes of ciliopathy patients might be attributed to defective paracrine signaling (1), including polydactyly due to defective sonic hedgehog (Shh) signaling (2) and renal cysts attributed to unbalanced Wnt signaling (3). Some data have raised the possibility that a fraction of these defects, especially Wnt, are driven by nonciliary functions of ciliary and basal body proteins $(2,4)$; other findings have indicated that the cilium/basal body Wnt roles are likely specific to discrete spatiotemporal contexts (5-9).

Although basal body and ciliary proteins are not signaling molecules per se, these structures are thought to operate as a hub for coordinating networks of signaling cascades. Components of various signaling pathways localize to basal body and cilium (10-13). Moreover, mutations in a single basal body or ciliary gene can lead to defects in more than one signaling pathway $(11,14)$, while loss-of-function mutations in signaling molecules such as the Shh regulator kinesin family member 7 (KIF7)

Authorship note: Yangfan P. Liu, I-Chun Tsai, Manuela Morleo, and Edwin C. Oh contributed equally to this work.

Conflict of interest: The authors have declared that no conflict of interest exists.

Note regarding evaluation of this manuscript: Manuscripts authored by scientists associated with Duke University, The University of North Carolina at Chapel Hill, Duke-NUS, and the Sanford-Burnham Medical Research Institute are handled not by members of the editorial board but rather by the science editors, who consult with selected external editors and reviewers.

Citation for this article: J Clin Invest. 2014;124(5):2059-2070. doi:10.1172/JCI71898
$(15,16)$ and the Wnt/planar cell polarity (PCP) effector Fritz (17) cause ciliopathies in some families.

The increasing association of ciliary and basal body proteins with signaling defects led us to ask whether a common mechanism might account for the convergence of multiple pathways. One candidate, the ubiquitin-proteasome system (UPS), is attractive for three reasons. First, the basal body is known to be a proteolytic center (18-21). Second, we reported previously that disruption of some basal body proteins results in loss of proteasomedependent degradation of $\beta$-catenin (5), a phenotype reproduced subsequently (22). Finally, we noted that, in addition to Wnt, proteasomal degradation is implicated in most paracrine signaling cascades known to be defective in basal body mutants. For example, in Notch-mediated lateral inhibition, a transmembrane ligand of the Delta-Serrate-LAG2 (DSL) family binds a transmembrane Notch receptor in the adjacent target cells (23). To reduce Notch signaling, DSL ligands are ubiquitinated, internalized, and degraded by the proteasome (24); similarly, the intracellular domain of the Notch receptor is degraded by the proteasome to reduce Notch signaling (25). Likewise, when Shh ligand is present, glioma-associated oncogenes 2 and 3 (GLI2/3) exist in their full-length activator forms, whereas they are truncated by proteasome-mediated proteolysis to their repressor forms when Shh is removed (26); both the activator and repressor forms of GLI2/3 are also degraded by the proteasome (27). Besides GLI2/3, suppressor of fused homolog (SUFU), a negative regulator of Shh signaling that physically localizes at the cilium, is also degraded in a proteasome-dependent manner (28). 
Given these observations, we wondered whether basal body and ciliary proteins might regulate multiple signaling pathways by controlling proteasome-mediated degradation of signaling mediators. We show that suppression of basal body-localized ciliopathy proteins led to defective proteasomal degradation of such mediators, which in turn caused dysfunction in three major cilia-associated signaling pathways (Shh, Wnt, and Notch) in vitro and in vivo. These observations are unlikely to reflect nonspecific cellular malaise; not only could the ciliopathy proteins tested here interact with specific regulatory subunits of the proteasome holoenzyme, but also depletion of each of Bardet-Biedl syndrome 4 (BBS4) and oral-facial-digital syndrome 1 (OFD1) selectively perturbed the subunit composition of the centrosomal proteasome. Further, signaling phenotypes due to depletion of BBS1, BBS4, and OFD1 could be rescued by activating proteasomal function. Finally, because the model predicts that other proteasome-dependent paracrine signaling pathways should also be defective in the absence of basal body proteins, we examined NF- $\kappa \mathrm{B}$ signaling, a pathway that has not been linked previously to basal body (dys)function. Consistent with our prediction, loss of each of BBS1, BBS4, and OFD1 repressed NF-אB activity, which could be restored by activating the proteasome. Taken together, these findings suggest that proteasome-mediated basal body regulation might be a common mechanism for a multitude of signaling cascades and that proteasomal activation might be a potential treatment paradigm for ciliopathies.

\section{Results}

Accumulation of signaling mediators upon depletion of ciliopathy proteins. We and others have reported previously that perturbation of some ciliopathy proteins alters the stability of Wnt signaling mediators such as $\beta$-catenin and Dishevelled $(5,8,21,29,30)$. To study the physiological relevance of these findings in vivo, we mated $B b s 4^{-/-}$ mice with a transgenic proteasome reporter mouse line expressing unstable ubiquitin-tagged GFP (31) to generate $U b^{G 76 V_{-}-G f p ~ B b s 4^{-/-}}$ mice. As a hallmark of potential proteasomal dysfunction, we examined GFP levels in a broad range of tissues with known pathology in BBS and observed no aberrant GFP accumulation in the kid-

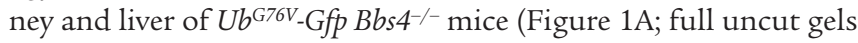
are shown in the Supplemental Material.) and modest increases in components of the central nervous system (hippocampus, cortex, and cerebellum; Figure 1A). By contrast, we detected significant accumulation of GFP from P14 onward in the retinas of $U b^{G 76 V-G f p}$ $B b s 4^{-/}$mice (Figure 1B). To identify which cell types were accounting for the GFP signal, we performed immunohistochemical analyses of retina from three $U b^{G 76 V_{-} G f p ~ W T ~ a n d ~ t h r e e ~} U b^{G 76 V}-G f p B b s 4^{-/}$ mice (masked to the genotypes). Cryosections from $U b^{G 76 V}$-Gfp $B b s 4^{-/}$mice revealed significant aggregation of GFP in the rod and cone photoreceptor layers consisting of the outer segment, inner segment, and outer nuclear layer (Figure 1C). This phenotype was restricted to photoreceptors (Figure 1C) and was unlikely to be secondary to retinal degeneration, as we observed no anatomical photoreceptor defects on P14, consistent with reported findings for other Bbs mouse mutants (29).

Given the specificity of GFP degradation defects in the photoreceptors of $B b s 4^{-/-}$mice and previous data on the abnormal stability of $\beta$-catenin in BBS4-depleted cells (5), we next asked whether proteasomal dysfunction might be more broadly relevant to basal body-mediated paracrine signal regulation. If this is true, then: (a) other basal body proteins should have similar functions; and (b) other known basal body-mediated pathways should also be perturbed. To evaluate functional links between basal body proteins, we first conducted an in silico study to analyze a network of coexpressed basal body and ciliary genes through a publicly available online tool (http://netview.tigem.it), which reconstructs the regulatory interactions among genes from genome scale measurements of gene expression profiles (32). This analysis revealed a subset of centrosomal transcripts $\left(P=3.5 \times 10^{-7}\right)$ including BBS1, BBS4, and OFD1 (Supplemental Figure 1; supplemental material available online with this article; doi:10.1172/JCI71898DS1). We thus considered the possibility that loss of OFD1 also leads to changes in the stability of signaling mediators.

We turned to an established cilium-dependent paracrine signaling pathway, Hedgehog signaling (Hh) (2), and developed an in vitro neural differentiation model, in which the embryonic WT murine stem cells (WTESs) and Ofd1-deficient murine embryonic stem cells (KOESs) were cultured to generate neural progenitors (Supplemental Figure 2), a model that recapitulates the multi-step process of neural development in embryos (33). KOESs are male murine cells containing a gene trap mutation in $O f d 1$. As $O f d 1$ is located on the $\mathrm{X}$ chromosome, $\mathrm{Ofd1}$ KOESs are hemizygous for $O f d 1$ and do not produce the protein (34). Comparing $O f d 1^{W T}$ neurons derived from WTESs and $O f d 1^{K O}$ neurons from KOESs, we found a significant increase in GLI2FL (full-length GLI2) and SUFU at the T8 time point in $O f d 1$-depleted neurons (Figure 2A). We also found increased levels of GLI2FL and SUFU in lysates from $O f d 1^{\Delta 4-5 /+}$ heterozygous female mouse embryos (data not shown) and $O f d 1^{\Delta 4-5 / y}$ hemizygous male mouse embryos (Supplemental Figure 3A) compared with levels observed in WT littermates (Figure 2B). In addition, GLI3FL (full-length GLI3) increased both in $O f d 1^{K O}$ neurons (Figure 2A) and in $O f d 1^{\Delta 4-5 /+}$ (data not shown) and $O f d 1^{\Delta 4-5 / y}$ mutant embryos (Figure $2 \mathrm{~B}$ ), with a concomitant decrease in glioma-associated oncogene 3 repressor (GLI3R) levels (Figure 2, A and B, and Supplemental Figure $3, \mathrm{~B}$ and $\mathrm{C})$.

Similar to Hh and Wnt signaling, Notch signaling is regulated through the cilium (13), and if the overarching hypothesis is correct, this pathway should also be perturbed in our mutants. As a first test, we cotransfected Flag-tagged Notch1 intracellular domain (NICD) into HEK-293-FT cells depleted of BBS4 by shRNAmediated gene silencing ( $p$ SuperBBS4) (5). Immunoblot analysis of transfected cells revealed an elevation of NICD protein levels upon knockdown of BBS4 (Figure 2C). Further, we asked whether loss of BBS4 modulates the protein levels of a DSL ligand, Jagged 1 (JAG1). We therefore assayed GFP-tagged JAG1 in BBS4-depleted and control cells. Similar to our findings with NICD, knockdown of BBS4 also resulted in an elevation of GFP-JAG1 (Figure 2D).

Selective disruption of UPS caused by loss of ciliopathy proteins. Given our data on Wnt, Shh, and Notch and the fact that the processing of components from these paracrine signaling pathways is known to be mediated by the proteasome $(11,35)$, we asked whether proteasomal agonists could ameliorate the signaling phenotypes of basal body mutants.

The expression levels of three catalytic subunits of the proteasome, proteasome subunit $\beta$ types 5, 6, and 7 (PSMB5, PSMB6, and PSMB7), as well as the corresponding peptidase activities, increase upon treatment with sulforaphane (36) [SFN; 1-isothiocyanato-4(R)-methylsulfinylbutane], an isothiocyanate extracted from cruciferous vegetables, rendering this compound an attractive initial substrate for our studies. We found that treatment with SFN rescued the accumulation of GLI2FL, 
A

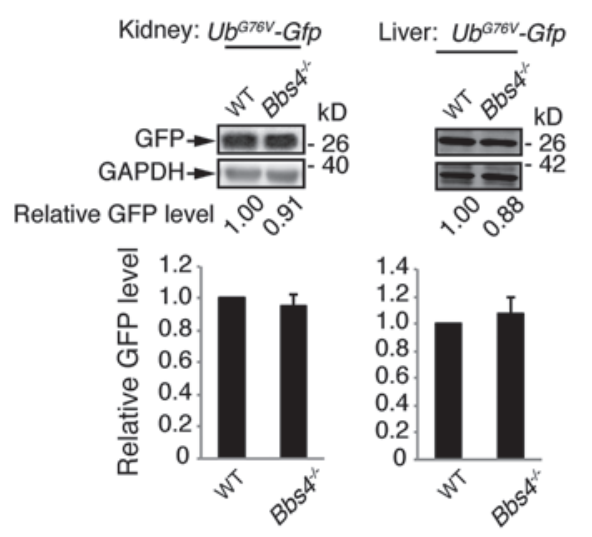

B
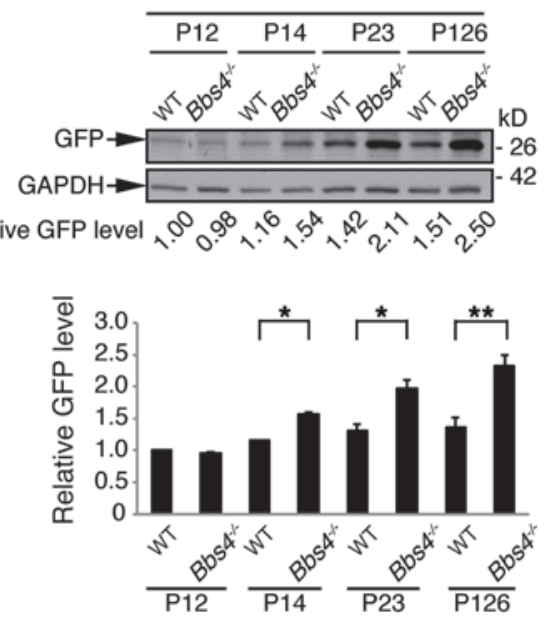
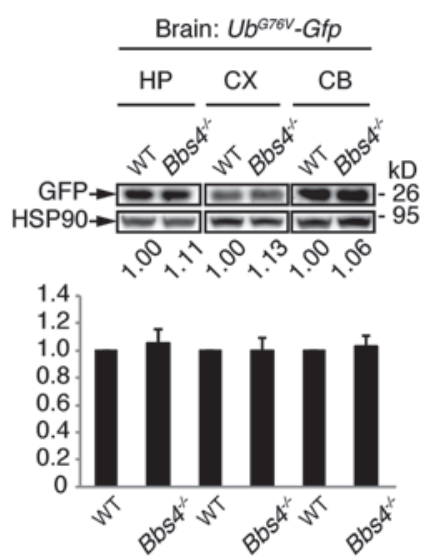

C

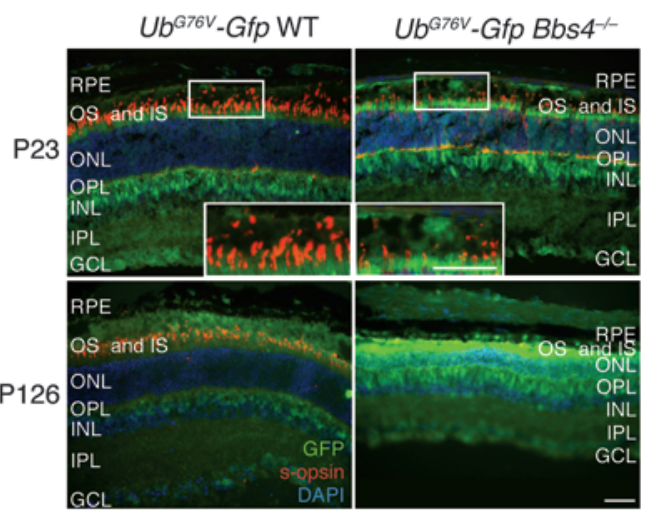

upon overexpressing our proteins of interest. We therefore overexpressed BBS4 in cells and observed a depletion of both NICD (Figure 3C and Supplemental Figure 3E) and JAG1 (Supplemental Figure $3 \mathrm{~F}$ ); further, we treated BBS4-overexpressing cells with two proteasome inhibitors, $N$-carbobenzoxyl-Lleucinyl-L-leucinyl-Lnorleucinal (MG132) and lactacystin as well as DMSO vehicle as a control. While cells overexpressing BBS4 showed a $21 \%-50 \%$ reduction in total NICD levels (Figure 3C and Supplemental Figure $3 \mathrm{E}$ ) and an approximately $50 \% \pm 16 \%$ reduction in total JAG1 levels (Supplemental Figure 3F), treatment with MG132 and lactacystin restored NICD protein levels to $93 \%$ (Figure 3C) and 138\% (Supplemental Figure 3E) of basal levels, respectively, and restored JAG1 levels to $99 \% \pm$ $38 \%$ of basal levels (Supplemental Figure 3F), suggesting that BBS4 can facilitate the degradation of Notch signaling mediators in a proteasome-dependent manner.

Regulation of the proteasome complex by ciliopatby proteins. Although our observations in mouse mutants, zebrafish embryos (5), and cultured cells argue that the basal body proteins we studied are responsible for regulating the proteasome, the mechanism of that action is unclear, leaving the possibility that the observed phenotypes are surrogate effects of generalized

GLI3FL, and SUFU in T8-derived Ofd1 ${ }^{K O}$ neurons (Figure 3A). Consistent with the participation of the proteasome in both the processing and degradation of GLI2/3, treatment with SFN reduced GLI3R protein levels (Figure 3A and Supplemental Figure 3D). Moreover, SFN treatment of cells depleted of BBS4 also revealed a reduction in $\beta$-catenin protein levels relative to vehicle-treated cells (Figure 3B), suggesting that defects in proteasome-dependent processing and degradation of GLI2/3, SUFU, and $\beta$-catenin can be ameliorated.

Taken together, our data argue for a role of basal body proteins in regulating proteasome-mediated degradation and that we should observe a reciprocal signaling/proteasomal phenotype cellular "malaise." We therefore explored the possibility of direct biochemical relationships between BBS1, BBS4, and OFD1 and the proteasome.

First, we conducted an unbiased mass spectrometry screen to identify putative OFD1-interacting proteins. This experiment uncovered a spectrum of proteasomal subunits including regulatory proteasome ATPase subunit 6 (RPT6), a finding confirmed by semiendogenous coimmunoprecipitation (Figure 4A). Independently, we tested for protein-protein interactions between multiple BBS proteins (BBS1, 2, 4, 5, 6, 7, 8, and 10) and several proteasomal subunits (proteasome subunit $\beta$ type 1 subunit in the 20S particle [PSMB1], regulatory proteasome non-ATPase subunit 


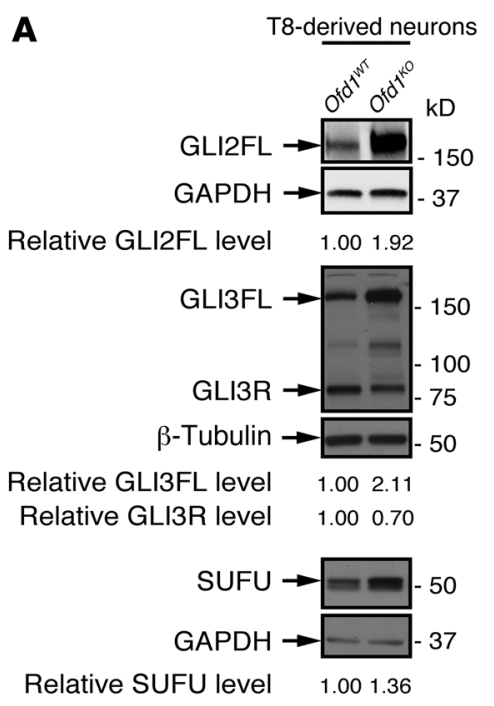

C

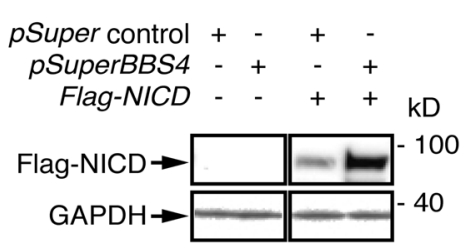

Relative NICD level N/A N/A 1.003 .51
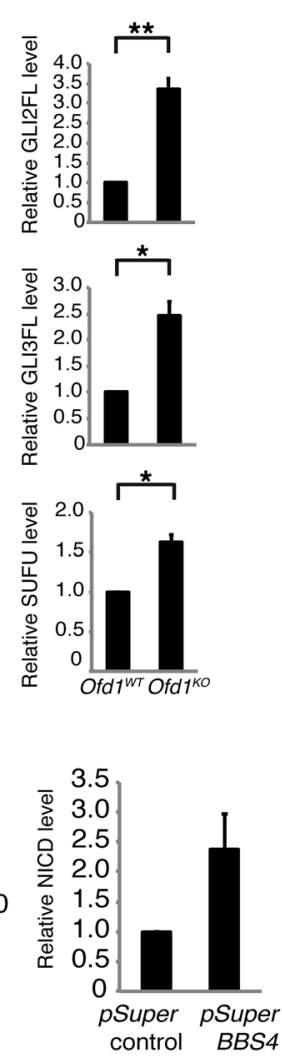

B
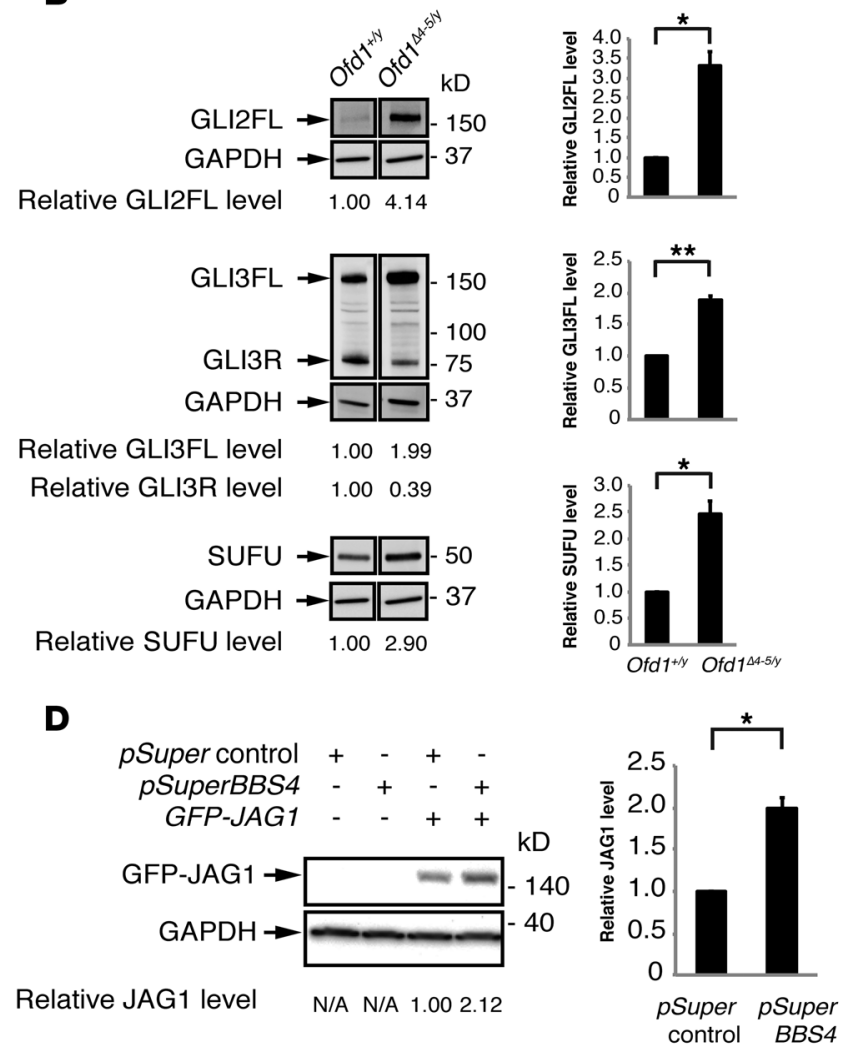

\section{Figure 2}

Accumulation of Shh and Notch signaling mediators upon depletion of ciliopathy proteins. (A) Accumulation of GLI2FL, GLI3FL, and SUFU as well as reduction of GLI3R in T8-derived Ofd $1^{\mathrm{KO}}$ neurons. (B) At E10.5, accumulation of GLI2FL, GLI3FL, and SUFU as well as reduction of GLI3R were detected in protein lysates from Ofd1 $144-5 / y$ mice, with Ofd1+/y mice used as controls. (C) Suppression of BBS4 increases Flag-tagged NICD levels compared with those in pSuper controls. (D) Suppression of BBS4 led to a 2-fold increase in GFP-tagged JAG1. The samples in each panel in $\mathbf{B}$ and $\mathbf{C}$ were run on the same gel but were noncontiguous. Bar graphs showing the SEM are plotted adjacent to each blot. ${ }^{\star} P<0.05$; ${ }^{*} P<0.01$.

10 [RPN10], regulatory proteasome non-ATPase subunit 13 [RPN13], RPT6, and non-19S regulatory subunit [PA28 $\gamma$ ], selected based on antibodies available for subsequent experiments). Under stringent detergent conditions (1\% Triton X-100), we confirmed the previously reported interaction between BBS4 and RPN10 (5) by semiendogenous coimmunoprecipitation (Figure $4 \mathrm{~A}$ ) and also observed a biochemical interaction of some BBS proteins (BBS1, BBS2, BBS4, BBS6, BBS7, and BBS8) with proteasomal components, while BBS5 and BBS10 did not interact with any tested proteasomal subunits (Supplemental Figure 4A). To test the physiological relevance of a BBS-proteasome interactome, we determined that BBS1 and RPN10 interacted at endogenous levels in protein lysates isolated from C57BL/6 mouse testes (Figure 4A).

To probe the relevance of these interactions, we asked whether loss of OFD1 or BBS4 alters the composition of the proteasome with respect to its subunits. Using a stable cell line expressing tagged regulatory proteasome non-ATPase subunit 11 (RPN11) (37), we suppressed OFD1, purified 26S proteasome complexes, and observed a robust reduction $(60 \% \pm 8 \%)$ of $26 \mathrm{~S}$-bound regulatory proteasome ATPase subunit 2 (RPT2) in OFD1-depleted cells in comparison with that in control, while total RPT2 protein abundance was not affected by OFD1 depletion (Figure 4B). We also assayed the fraction of 26S-bound RPN10 in BBS4-depleted cells and observed a modest but significant reduction $(20 \% \pm 3 \%)$ in RPN10 (Supplemental Figure 4B). Furthermore, we also tested the localization of RPN10 around the centrosome in HEK-293 cells depleted of BBS4 and OFD1 by quantifying the RPN10 signal that localized around centrosomal $\gamma$-tubulin; we did not observe appreciable changes in BBS4-depleted cells (data not shown), possibly because of a lack of spatial resolution. By contrast, we observed a $43 \% \pm 2 \%$ reduction of pericentriolar RPN10 levels (normalized to cytoplasmic RPN10 levels) in OFD1-depleted cells (Figure 4C). Given these data, we tested whether loss of OFD1 or BBS4 altered the composition of RPN10, RPT2, and other proteasomal components by sedimentation. Based on the commercial availability of reliable antibodies against proteasomal subunits, we found that RPN10, RPN13, RPT2, and RPT6 were enriched in $\gamma$-tubulinenriched fractions in control cells (Figure 4D and Supplemental Figure 4C). Depletion of OFD1 or BBS4, however, resulted in a shift in the peak expression of the proteasomal subunits (Figure 4D and Supplemental Figure 4C). Taken together, these data suggest that BBS proteins and OFD1 regulate the composition of the centrosomal proteasome, likely through direct biochemical interactions.

Activation of proteasomal components ameliorates signaling defects caused by loss of ciliopathy proteins that reside at the basal body. Our observations next led us to speculate that (a) if reduction of proteasomal 

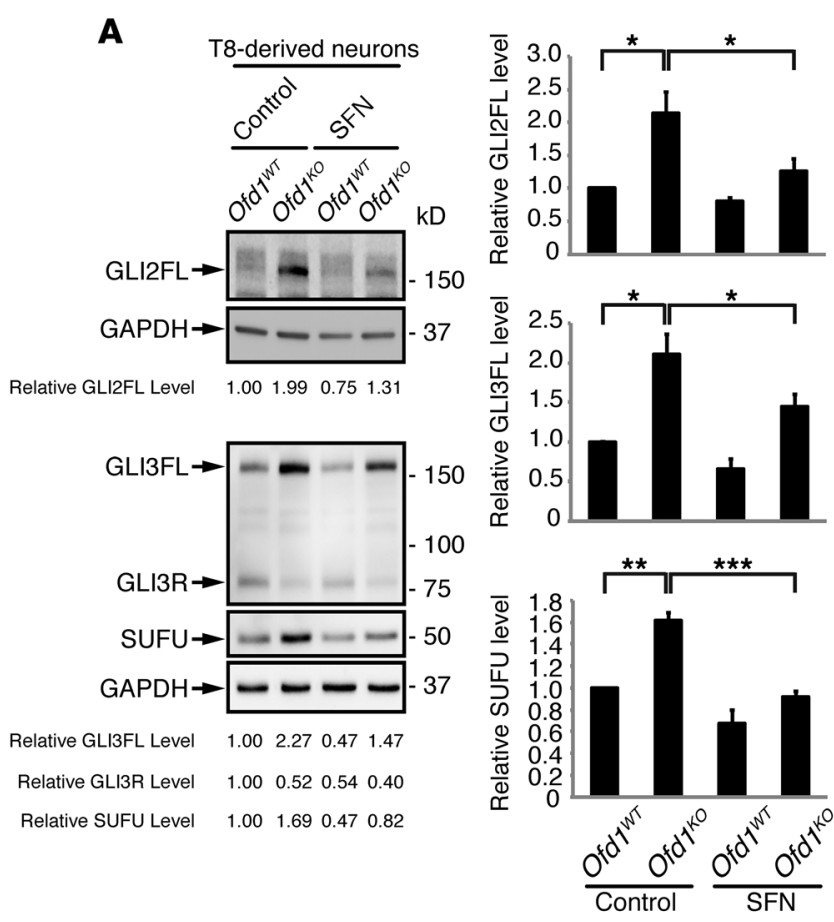
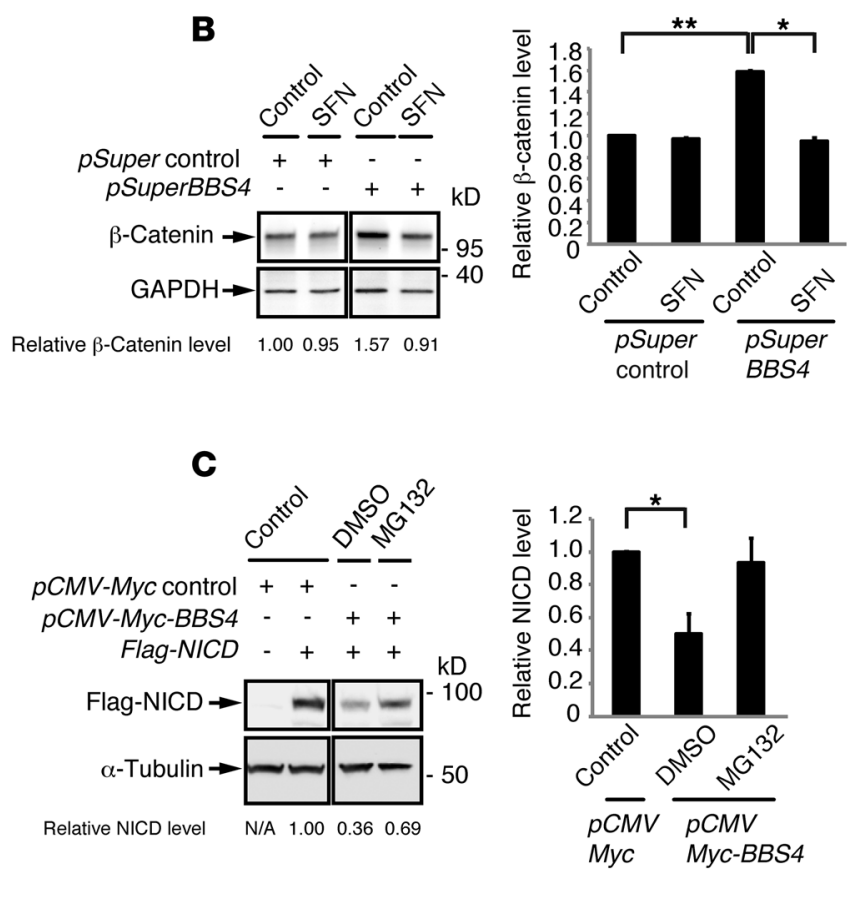

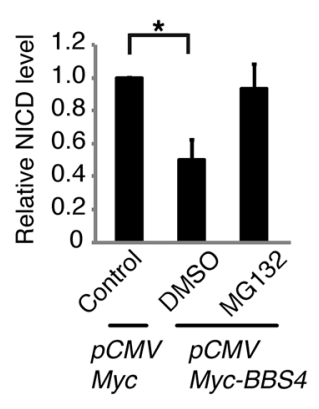

\section{Figure 3}

Disruption of proteasomal degradation caused by loss of ciliopathy proteins. (A) Treatment of proteasomal agonist SFN ameliorated the accumulation of GLI3FL, GLI2FL, and SUFU in T8-derived Ofd1 $1{ }^{K O}$ neurons. (B) Suppression of BBS4 in HEK-293-FT cells led to a 1.57-fold increase in $\beta$-catenin protein levels that could be rescued by SFN. (C) Overexpression of BBS4 reduced NICD levels. MG132 treatment restored Flag-NICD levels. Samples in each panel in $\mathbf{C}$ were run on the same gel but were noncontiguous. Bar graphs showing SEM are plotted adjacent to each blot. ${ }^{\star} P<0.05 ;{ }^{* \star} P<0.01 ;{ }^{* * *} P<0.001$.

subunits in the centrosomal fraction of BBS4- or OFD1-depleted cells results in hampered degradation of signaling mediators, then increasing expression of the corresponding proteasomal subunits may ameliorate defective signaling phenotypes; and (b) activation of the proteasome might compensate for signaling defects.

We and others have reported previously that loss of ciliopathy proteins produces defects in convergent extension (CE) movements during gastrulation in zebrafish embryos $(5,29,30)$. In addition to and independent of these phenotypes, we observed defects in the definition of somite boundaries in zebrafish embryos injected with morpholinos against bbs genes (bbs morphants [bbs MO]) (Supplemental Figure 5A and ref. 38). In vertebrates, somites develop as epithelial blocks at a temporal and spatial periodicity that is controlled, in part, by Notch signaling (39). Given the phenotypic overlap between $b b s$ morphants and segmentation mutants (mib, bea, des, and aei) (39), as well as our biochemical Notch observations in BBS4-deficient cultured mammalian cells, we wondered whether dysfunction of these proteins might influence Notch signaling. Upon injection of the established bbs4 morpholino $(5,38)$ into 1- to 8-cell-stage embryos, and scoring at the $9 \pm 1$ somite stage ( $9 \pm 1$ ss), $92 \%$ of embryos exhibited an expansion of the anteroposterior midline expression domain of her4 (Supplemental Figure 5B), whose transcription is targeted directly by notch1 activation (40). To differentiate between ectopic expression at developmental stages, when Notch signaling is already active, and the possibility that signaling is persistent throughout stages when it should be diminished, we performed an incremental developmental series of her 4 in situ hybridization over the first 5 days of development. Scoring WT embryos (staged by number of somites and, later, by the presence of anatomical features such as the swim bladder to ensure that embryos of the same age were compared across experiments), we observed that the expression of her 4 in neural structures of the head, including the developing forebrain, midbrain, hindbrain, and eye, was robust through 2.5 days post fertilization (dpf) and then began to wane. In bbs 4 morphants, we saw persistent her 4 expression through $5 \mathrm{dpf}$, especially in the eye (Supplemental Figure 5C).

Given the availability of experimentally tractable signaling phenotypes, we asked whether overexpression of proteasomal subunits that were seen to mislocalize in BBS4- and OFD1-depleted cells might rescue the bbs 4 and ofd 1 morphant phenotype. Upon blind scoring for the effects of coinjecting human RPN10, RPN13, or RPT6 mRNA with the bbs 4 or ofd 1 morpholino, we measured substantial rescue of defective $\mathrm{CE}$, somitic definition, and persistent her4 expression (Figure 5A).

Next, as an independent test, we examined bbs 4 morphants coinjected with SFN, a known transcriptional activator of proteasomal subunits (36). Upon blind scoring at the $9 \pm 1$ ss, there was a significant reduction of $b b s 4$ morphants with $\mathrm{CE}$ and somitic defects $(P<0.001$; Figure $5 \mathrm{~B})$. Moreover, coinjection of SFN with a bbs4 morpholino ameliorated the ectopic expression of her 4 in neural structures at $4.5 \mathrm{dpf}$ (Figure 5B). We also observed a similar rescue of bbs 1 and ofd 1 morphants, in which coinjection of SFN gave rise to the same robust rescue of defective CE phenotypes, somitic defects, and persistent her 4 expression as that observed in bbs4 loss-of-function models (Figure 5, C and D). 
A
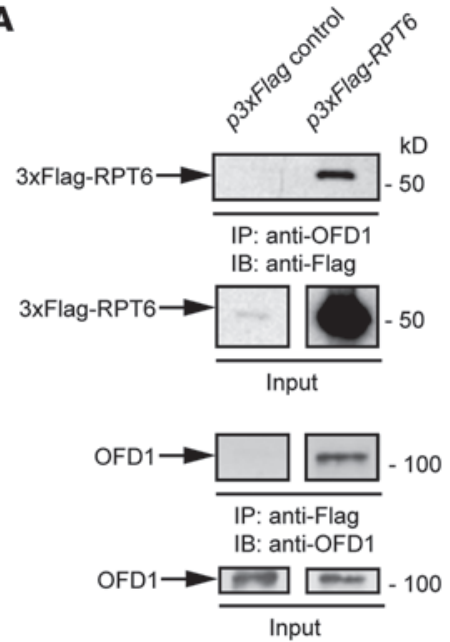

Input

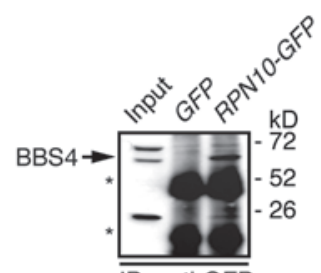

IP: anti-GFP

IB: anti-BBS4

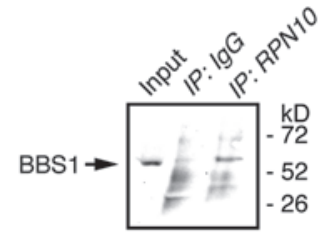

IP: anti-RPN10 IB: anti-BBS1

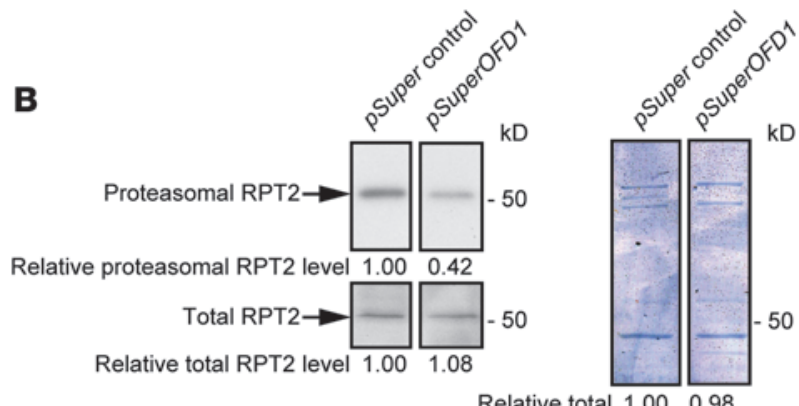

Relative total $1.00 \quad 0.98$

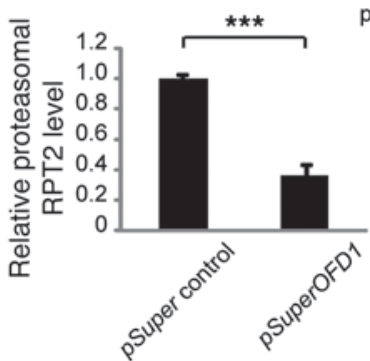

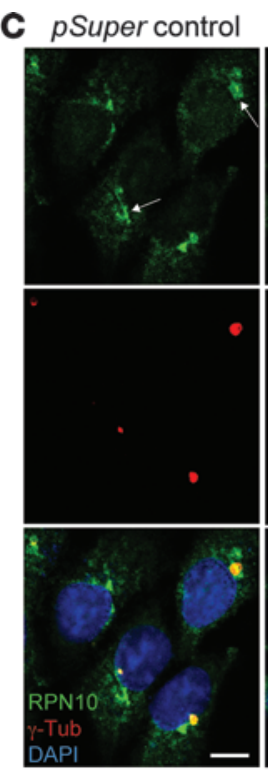

pSuperOFD1
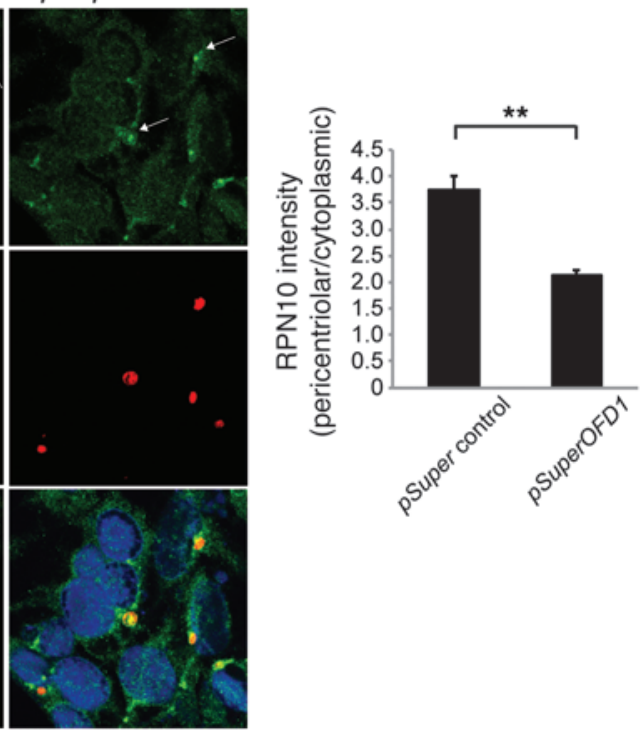

\section{Fraction}

\begin{tabular}{llllllll}
\cline { 2 - 7 } & & & & & & &
\end{tabular}
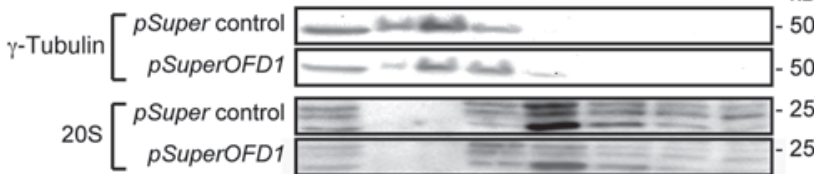

PA28 $\left[\begin{array}{c}p \text { Super control } \\ \text { pSuperOFD1 }\end{array}-35\right.$

RPN10 $\left[\begin{array}{c}p \text { Super control } \\ p \text { SuperOFD1 }\end{array}-50\right.$
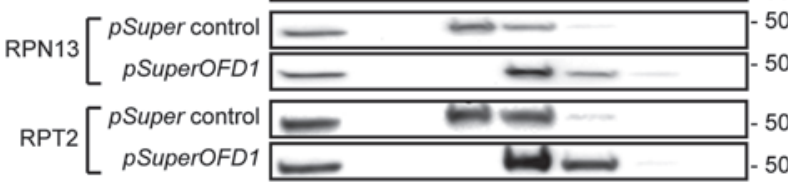

RPT6 $\left[\begin{array}{c}p \text { Super control } \\ \text { pSuperOFD1 } \square-50\end{array}\right.$

Sucrose gradient

\section{Figure 4}

Ciliopathy proteins interact with proteasomal components and regulate proteasome composition. (A) Immunoblots show interaction between endogenous OFD1 and Flag-tagged RPT6 and endogenous BBS4 and GFP-tagged RPN10. An endogenous interaction was detected between BBS1 and RPN10 from protein lysate isolated from the testis of C57BL/6 mice. (B) Immunoblots show that suppression of OFD1 in HEK-293 cells reduced RPT2 protein levels in purified $26 \mathrm{~S}$ proteasome. Densitometry measurements of proteasomal RPT2 protein levels were plotted $(n=3)$, and no significant difference in total RPT2 abundance was detected. Coomassie blue staining were carried out as a loading control and to measure the efficiency of the $26 \mathrm{~S}$ proteasomal purification process. (C) Suppression of OFD1 in HEK-293 cells resulted in the reduction of pericentriolar RPN10 levels (normalized to cytoplasmic RPN10 levels) in OFD1-depleted cells. Scale bar: 10 um. (D) HEK-293-FT cells transfected with pSuper control and pSuperOFD1 were subjected to sucrose gradient centrifugation. Fractions (fractions 6-12 of 13 fractions) were then analyzed by immunoblotting with antibodies against $\gamma$-tubulin and proteasomal subunits. In control cells, the fractions enriched with all proteasome subunits partially overlapped with fractions enriched with $\gamma$-tubulin (fraction 8), and when OFD1 was depleted, peak levels of RPN10, RPN13, RPT2, and RPT6 shifted significantly and resulted in a decrease in the overlap between $\gamma$-tubulin-enriched fractions and 19S subunit-enriched fractions. ${ }^{\star \star} P<0.01 ;{ }^{* \star} P<0.001$.

To substantiate the rescue effects of SFN, we used another proteasomal agonist, mevalonolactone [known as mevalonic acid lactone, mevalonate, and $( \pm)$ - $\beta$-hydroxy- $\beta$-methyl- $\delta$-valerolactone and abbreviated hereafter as MVA], to ask whether broad activation of the proteasome can alleviate basal body-dependent phenotypes. We coinjected MVA with the bbs4 morpholino into zebrafish embryos, and upon blind scoring at $9 \pm 1$ ss, we found a reduction in bbs 4 morphant zebrafish with CE defects from $47.8 \%$ to $11.4 \%$ with MVA (Supplemental Figure 6). To investigate the persistence of Notch signaling in bbs4 morphant zebrafish coinjected with MVA, we performed in situ hybridization for her4. Coinjection of MVA with a bbs4 morpholino reduced her4 
expression levels in neural structures, especially the retina, to those in WT zebrafish by $4.5 \mathrm{dpf}$ (Supplemental Figure 6).

$N F-\kappa B$ signaling defects in basal body ciliopathy mutants can be rescued by activation of the proteasome. Our findings indicate that Wnt, Notch, and Shh phenotypes generated upon loss of three basal body proteins might converge at the point of proteasomal degradation. We therefore considered a model in which the basal body region serves as a broad regulator of paracrine signaling through proteasomal degradation. If this model is true, then (a) other paracrine pathways not implicated previously in ciliary/basal body biology, but known to be regulated by the proteasome, should exhibit signaling defects upon depletion of BBS proteins or OFD1; and (b) the model should also be able to predict the direction of the phenotype (suppressed or excessive signaling). To test this, we turned to NF- $\mathrm{KB}$ signaling, a pathway involved in inflammatory responses and lymphoid organogenesis with no known link to basal body (dys)function. In response to stimuli, IкBs are degraded by the proteasome, releasing NF- $\mathrm{KB}$ to translocate to the nucleus and activate the transcription of target genes (41).

We transfected HEK-293-FT cells with an NF-кB luciferase reporter plasmid containing three copies of the $\kappa \mathrm{B}$ response elements of the murine MHC class I promoter (3X-кB-L). Cells stimulated by TNF- $\alpha$ and cotransfected with the $p$ SuperBBS4, pSuperBBS1, and $p$ SuperOFD 1 plasmids displayed a $55 \%, 53 \%$, and $72 \%$ reduction in NF- $\kappa \mathrm{B}$ activity, respectively, compared with that of control cells; incubation with SFN for 6 hours restored NF- $\kappa B$ activity (Figure 6, $\mathrm{A}-\mathrm{C}$ ). In cells with depleted basal body proteins, the direction of the NF- $\mathrm{KB}$ activity change (suppressive signaling) is opposite that of Wnt and Notch activity change (excessive signaling) $(5,22)$. This inverse relationship is consistent with our model, since the predicted basal body-regulated proteasomal degradation substrates are IкBs, which are negative regulators, while $\beta$-catenin and NICD are positive regulators. Also consistent with the luciferase assays, we observed accumulation of GFP-tagged IкB $\beta$ in BBS4-, $B B S 1$-, or OFD1-suppressant cells, which can also be ameliorated by SFN treatment (Figure 6, D and E).

Finally, to probe the potential physiological relevance of the observed NF-KB data in vivo, we examined the expression and protein levels of the proteasomal substrate I $\mathrm{B} \beta \beta$ in mice lacking Ofd1. Immunoblot analysis showed no differences in IкB $\beta$ protein levels on protein lysates from E10.5 Ofd $1^{\Delta 4-5 / y}$ mutant embryos and controls (data not shown). Since loss of $O f d 1$ in mice results in prenatal lethality, we generated and crossed Ofd1-floxed mice $\left(O f d 1^{f l}\right)$ with a $C A G$-Cre-ERTM-inducible general deletor line to examine I $\mathrm{B} \beta \beta$ protein levels in postnatal $O f d 1$ knockout mice. In Ofd $1^{f l / y} C A G-C r e-E R^{T M}\left(O f d 1^{f l y} C K O\right)$ mice, Ofd1 inactivation was achieved at E18.5 by tamoxifen injection, and renal cysts were not observed at P8 (precystic stage). By P20, we observed a replacement of the renal parenchyma by cysts (cystic stage). Immunoblot analysis revealed an accumulation of $I \kappa B \beta$ in protein lysates from kidney of $O f d 1^{f l / y} \mathrm{CKO}$ male mice compared with controls; crucially, this phenotype was evident in both precystic and cystic stages (Figure 6F), suggesting that the accumulation of IкB $\beta$ is not a by-product of tissue disorganization and cystogenesis. At the precystic stage, mRNA levels of the I $\mathrm{B} \beta$ gene (Nfkbib) were lower in $O f d 1^{f l / y}$ CKO mice compared with mRNA levels in WT animals (Figure 6G), confirming that the accumulation of IкB $\beta$ is not due to increased mRNA transcription levels. Nfkbib mRNA levels were comparable between $O f d 1^{f l y} \mathrm{CKO}$ and controls at the cystic stage (Figure 6G).

\section{Discussion}

The study of ciliary and basal body proteins has highlighted a complex role for this cellular region in the regulation of signaling pathways. These observations have raised critical questions, including whether dedicated signaling transduction machinery aggregate around the cilium and basal body. Several transduction components have been localized to the basal body and/or the ciliary axoneme, including Smoothened, GLI proteins, SUFU, $\beta$-catenin, adenomatosis polyposis coli (APC), and Notch3 $(8,10,11,13,29)$. At the same time, a model in which the cilium facilitates each pathway independently is difficult to reconcile with the fact that (a) with the possible exception of the PCP transducer Fritz $(17)$ and the Shh motor $\operatorname{KIF} 7(15,16)$, none of the other 60-plus genes and proteins mutated in human ciliopathies are components of a specific paracrine signaling pathway; (b) the phenotype of ciliopathy patients is a mixture of defects more consistent with a context-specific pathway dysfunction; and (c) animal models ablated for specific ciliary or basal body proteins exhibit multiple signaling defects (1).

Our data suggest a simpler model, in which at least some basal body proteins play a role in signal transduction regulation by exerting their primary effect not on a given pathway per se, but by regulating context-dependent proteolytic degradation. The alternative would be that the observed phenotypes are the nonspecific consequence of generalized cellular malaise and that the observed rescue effects were reflective of broad improvement in the ability of the cell to eliminate proteins targeted for degradation. Taken together, our experiments favor the former model. Cells and embryos suppressed or ablated for each of $B B S 1, B B S 4$, and OFD1 had defects in proteasomal clearance of both reporter proteins and specific signaling components that included $\beta$-catenin, NICD, JAG1, GLI2, GLI3, SUFU, and IкB $\beta$. It is also notable that the pronounced defects in proteasomal activity were concomitant with overt anatomical pathology in our murine models of disease, such as in the retina of Bbs mice and the cystic kidneys of conditional $O f d 1$ animals, whereas no GFP accumulation was observed in Bbs mutant kidneys that, in our colony, never exhibited cyst formation.

We tested the robustness of the model in four ways: (a) by predicting that NF- $\kappa \mathrm{B}$ signaling, which requires proteasomal degradation (41), but has no known ciliary link, would be defective in the absence of our proteins of interest; (b) by predicting the direction of the defect in NF-кB signaling; (c) by ameliorating the signaling defects for each tested pathway in vivo through the chemical upregulation of proteasomal components; and (d) by demonstrating that in the absence of some basal body proteins, the centrosomal proteasome is partially depleted of the various regulatory subunits whose overexpression also has an ameliorating defect in vivo.

Notably, we observed sucrose fraction sedimentation changes in multiple proteasomal subunits in the absence of OFD1 or BBS4, arguing that ciliopathy phenotypes are unlikely to be driven by specific defects in only one subunit, consistent with the observations that the mice haploinsufficient for the subunit RPN10 are phenotypically normal, at least by gross pathology, while homozygous Rpn10-/- mutants are embryonic lethal (42). An attractive mechanism is one in which basal body proteins regulate the composition of multiple subunits in the proteasome holoenzyme in a contextdependent manner. This is known to occur during cellular stress (43), and it is plausible that ciliary signaling can have a similar effect. 
A

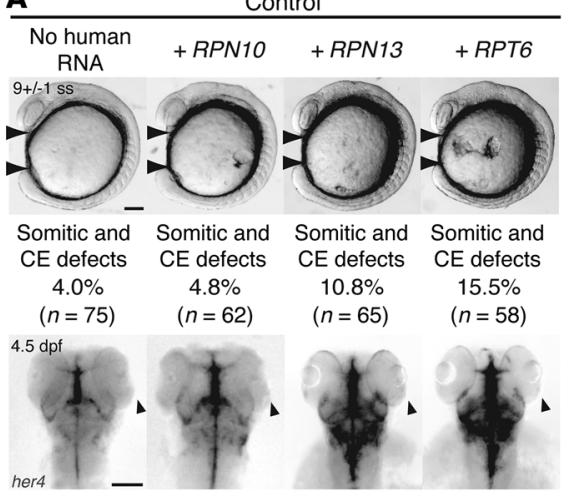

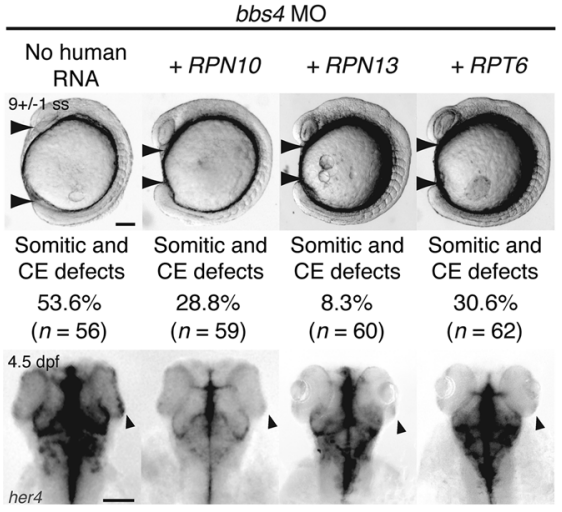

bbs $4 \mathrm{MO}$

bbs $4 \mathrm{MO}$
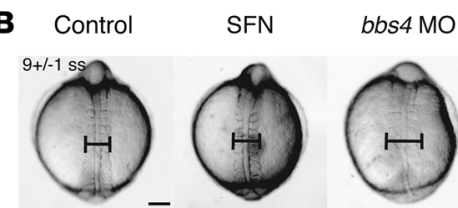

+ SFN
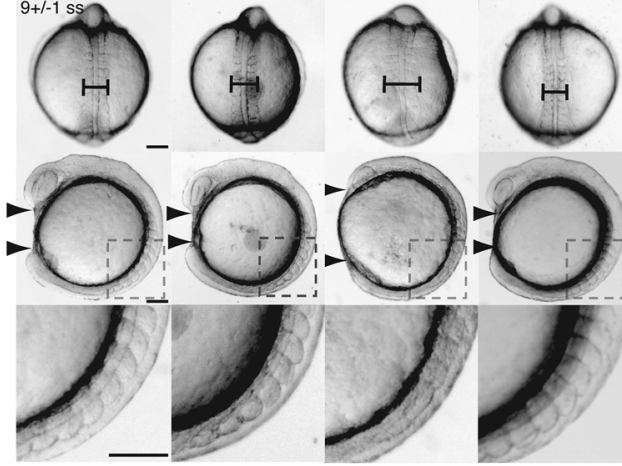
Somitic and Somitic and
CE defects

Somitic and CE defects
$7.4 \%$ $(n=47)$

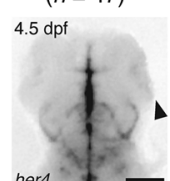

$(n=54)$

CE defects $53.3 \%$
$(n=45)$
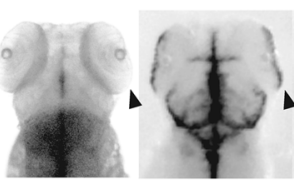

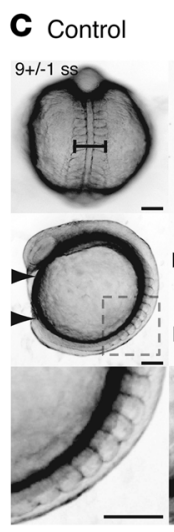

Somitic and Somitic and
CE defects
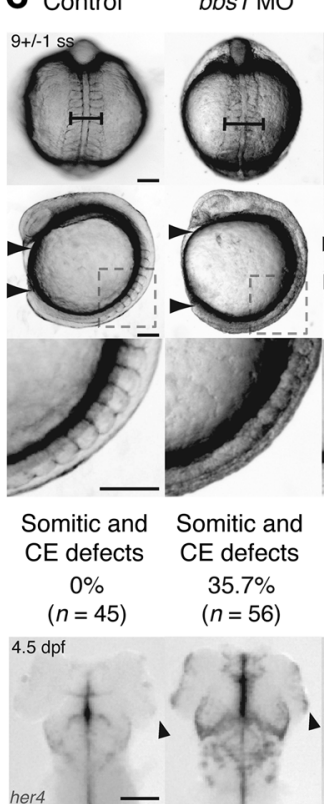

bbs $1 \mathrm{MO}$ $+\mathrm{SFN}$
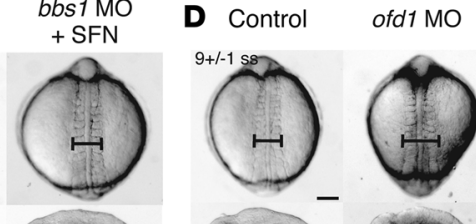

+ SFN
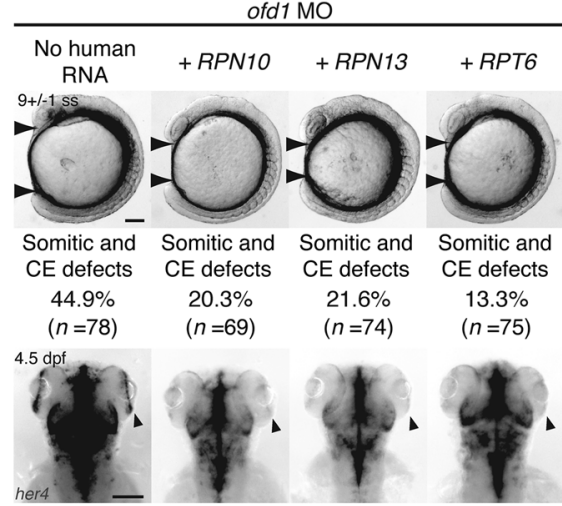

Figure 5

Activation of proteasome ameliorates signaling defects in bbs and ofd1 morphant zebrafish embryos. (A) Coinjection of human RPN10, RPN13, and RPT6 mRNA into bbs4 and ofd 1 morphant zebrafish embryos rescued somitic and CE defects at the $9 \pm 1$ ss and ectopic expression of her4 in the eye (arrowheads in lower row) at $4.5 \mathrm{dpf}$. CE defects were scored based on the body gap angle (arrowheads in upper row). Expression of her4 was detected by whole-mount RNA in situ hybridization. (B, C, and D) Coinjection of SFN rescued somitic and CE defects as well as ectopic her4 expression in bbs4 (B), bbs1 (C), and ofd1 (D) morphant zebrafish embryos, while injection of SFN alone did not give rise to any obvious phenotype (B). As shown in the top row (dorsal view), the somites (bars) were longer in bbs4 (B), bbs1 (C), and ofd1 (D) morphants and were shortened in morphant zebrafish embryos coinjected with SFN. In the second row (lateral view), the body gap angle (arrowheads) was greater in morphants and reduced in the presence of SFN. Dashed boxes delimit the enlarged images in the third row, showing the effects of SFN treatment on somite boundary definition defects. Percentage of embryos with somite boundary definition and CE defects and sample size $(n)$ are noted below the images of each condition. Scale bars: $100 \mu \mathrm{m}$.

Moreover, it is likely that proteasomal dysfunction is only one contributor to the pathology of ciliopathies. The BBS proteins have been proposed to perforate vesicles and regulate vesicular transport, possibly into the ciliary axoneme, in a GTPase-dependent fashion (44), while BBS4 has also been reported to bind to EXOC7 (45). These data intimate additional layers of complexity, in which these proteins can either have both promoting and inhibitory functions with regard to proteasomal targeting or other subcellular surveying mechanisms, or might be themselves inhibited or activated by other ciliary and axonemal proteins in a spatiotemporal context.
Emergent data suggest that the composition and function of primary cilia vary significantly across cell types (46). Such complexity might account for conflicting observations, in which each of the major signaling pathways mapped to date on this subcellular region have been shown to be activated, suppressed, or irrelevant in different tissues (4-6, 46, 47).

Finally, our findings indicate potential therapeutic applications. SFN has shown no toxic effects in humans and is currently being tested in several anticancer trials $(48,49)$. Should this compound show therapeutic benefit in rodent ciliopathy mod- 
A

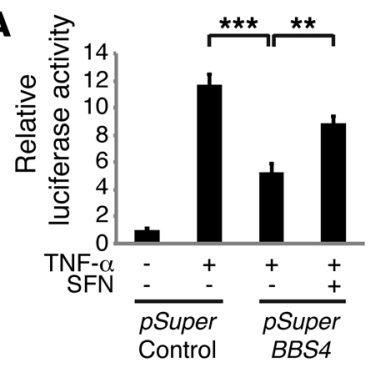

D

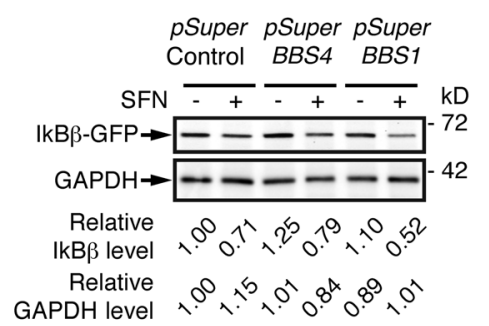

$\mathbf{F}$

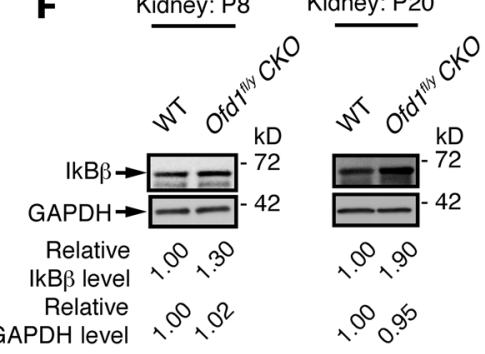

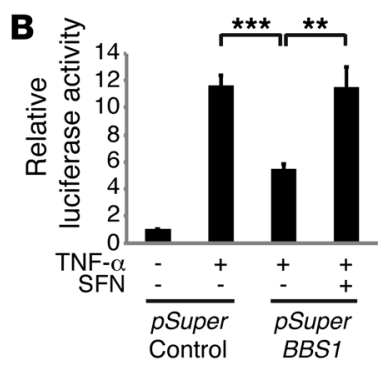
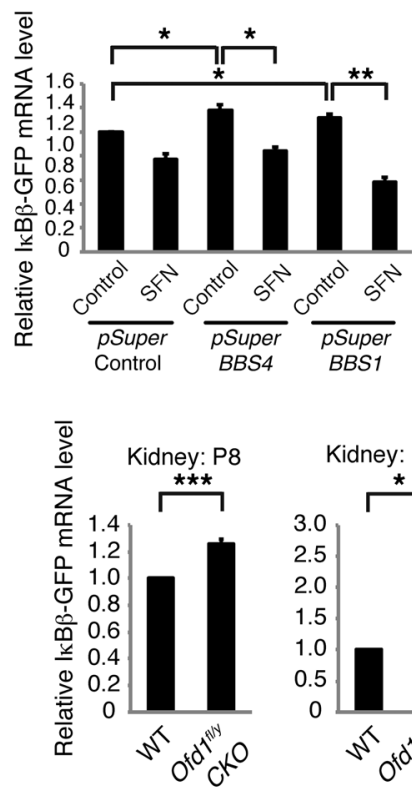
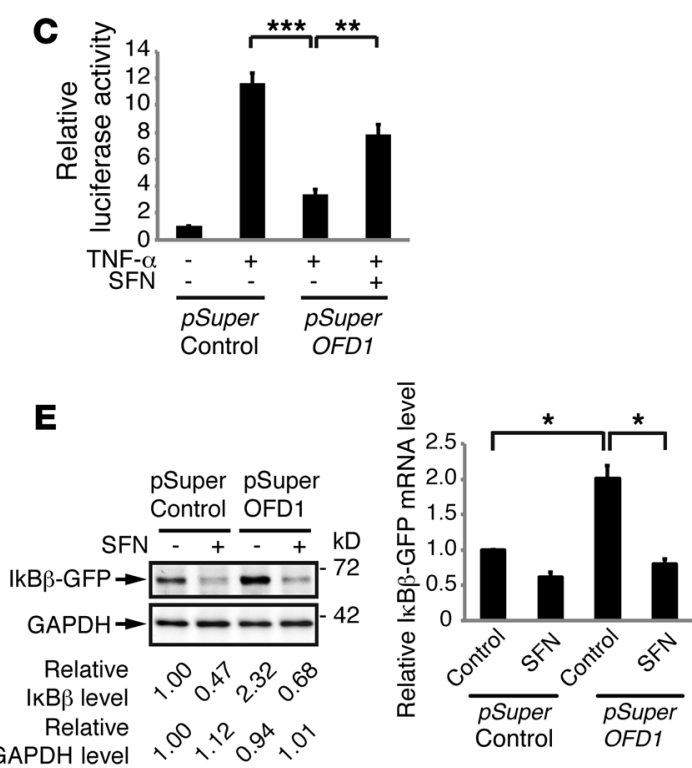

G

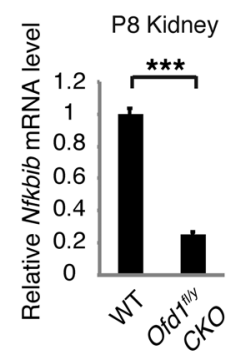

P20 Kidney

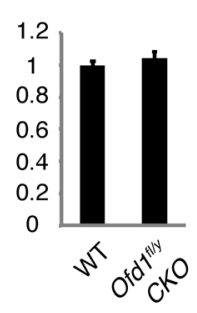

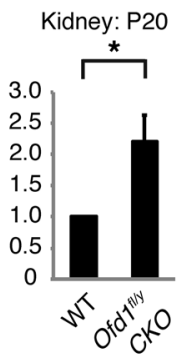

\section{Figure 6}

NF-KB signaling defects in BBS4-, BBS1-, and OFD1-depleted cells, and Ofd1 conditional knockout mice. (A-C) Suppression of BBS4 (A), BBS1 (B), or OFD1 (C) in HEK-293-FT cells led to decreased 3X-kB-L luciferase reporter responsiveness to a 12-hour TNF- $\alpha$ treatment. Incubation with SFN for 6 hours partially restored sensitivity to TNF- $\alpha$ stimulation $(n=3)$. (D and E) GFP-tagged I $\kappa \beta \beta$ protein levels were higher in HEK-293-FT cells depleted of BBS4, BBS1 (D), or OFD1 (E) compared with those in control. SFN incubation rescued IкB $\beta$-GFP accumulation. (F) Protein levels of IKB $\beta$ in kidney tissues isolated from Ofd $1^{f l y}$ CAG-Cre-ER ${ }^{T M}$ (Ofd $1^{f l l y} C K O$ ) mice at P8 (precystic stage) and P20 (cystic stage) were higher than the levels in kidney tissues from WT littermates. (G) mRNA levels of $/ \kappa B \beta$ (Nfkbib) in kidney tissues were lower in Ofd $1^{f l l y} C K O$ compared with mRNA levels in WT animals at P8 and were comparable between Ofd tlly CAG-Cre-ER $^{\text {TM }}$ and controls at P20 $(n=3)$. Bar graphs showing SEM are plotted adjacent to each blot. ${ }^{\star} P<0.05$; ${ }^{\star *} P<0.01$; ${ }^{\star \star \star} P<0.001$.

els, it would be a candidate for clinical trials in some ciliopathy patients. More broadly, other SFN derivatives as well as unrelated proteasome agonists might also represent natural candidates. However, SFN is likely to confer a partial benefit that is possibly both genotype and subphenotype dependent. First, the observed rescue of embryos injected with the compound, although significant, was not complete. Second, SFN is unlikely to be of benefit to all pathway defects. The proteasome is known to degrade the negative regulator SUFU and both the activator and the repressor forms of GLI2/3; as such, it is likely that downstream Shh signaling defects will not be rescued. Likewise, long-term exposure to SFN repressed NF- $\kappa \mathrm{B}$ activity (data not shown), suggesting that delivery, dosage, and duration of exposure might be critical. Finally, if basal body ciliopathy proteins participate in other cellular processes that regulate signaling, proteasomal modification will only be partially effective. Given our findings, however, a compound approach that includes targeting the proteasome may be of benefit.

\section{Methods}

Immunoblotting. Transfected cells and mouse tissues were lysed in modified RIPA buffer [150 mM sodium chloride, $50 \mathrm{mM}$ Tris-HCl, pH7.4, 1\% nonidet P-40, $0.1 \%$ sodium deoxycholate, $1 \mathrm{mM}$ EDTA] with $1 \times$ proteasome inhibitor (Roche) and centrifuged at $4^{\circ} \mathrm{C}$ for 15 minutes. Protein concentration was measured by Lowry assay using the DC Protein Assay Kit (Bio-Rad) on a DU 530 Life Science UV/Vis Spectrophotometer (Beckman Coulter). Total protein in each sample was separated by SDS-PAGE on $4 \%$ to $15 \%$ Mini-PROTEAN TGX Precast Gel (Bio-Rad) with a Spectra Multicolor Broad Range Protein Ladder (Fermentas) and transferred to an Immun-Blot PVDF Membrane (Bio-Rad). The membrane was blocked with $5 \%$ nonfat milk or $5 \%$ BSA (Sigma-Aldrich) and probed with the following commercial primary antibodies: anti-GAPDH (ab9484 from Abcam or sc-32233 from Santa Cruz Biotechnology Inc.); anti-GFP (sc-8334; Santa Cruz Biotechnology Inc. or ab13970; Abcam); anti-HSP90 (sc-7947; Santa Cruz Biotechnology Inc.); anti-GLI2 (AF3635; R\&D Systems); anti-GLI3 (AF3690; R\&D Systems); anti-SUFU (sc-10934; Santa Cruz Biotechnology Inc.); anti-Flag (F7425; Sigma-Aldrich); anti-catenin (sc-7199; Santa Cruz 
Biotechnology Inc.); anti- $\alpha$-tubulin (T6199; Sigma-Aldrich); anti-NICD (ab8925; Abcam); anti-hsOFD1 (rabbit polyclonal antisera against human full-length OFD1 NM_003611); anti-mmOFD1 (rabbit polyclonal antisera against a portion of murine OFD1 NM_177429 aa 461-884); anti-BBS4 (AB15009; Millipore); anti-BBS1 (a166613; Abcam); anti- $\gamma$-tubulin (T7451; Sigma-Aldrich); anti-20S (NB600-1016; Novus); anti-PA28 (NBP1-54587; Novus); anti-RPN10 (ab20239; Abcam); anti-RPN13 (H00011037-M01; Novus); anti-RPT2 (AP-107; Boston Biochem); anti-RPT6 (BML-PW9265; Enzo Life Sciences); anti-IкB $\beta$ (sc-9248; Santa Cruz Biotechnology Inc.); anti-Myc (m4439; Sigma-Aldrich); and anti-HA (ab16918; Abcam). Densitometric analysis was performed with Image Lab (Bio-Rad), Quantity One (Bio-Rad), or Image J 1.44p (NIH) software.

Immunohistochemistry. Mouse eyes were fixed in 4\% PFA, followed by immersion in sucrose (10\%, 20\%, and 30\%) in PBS. With the lens removed, eyecups were embedded in Optimal Cutting Temperature Compound (Sakura) and flash frozen. Cryosections were blocked with 10\% FBS in PBS and probed with primary antibodies anti-GFP (ab13970; Abcam) and anti-S-opsin (a gift from Jeremy Nathans, Johns Hopkins University, Baltimore, Maryland, USA), followed by secondary antibodies Alexa Fluor 488 IgG (Invitrogen) and Alexa Fluor 594 IgG (Invitrogen). Nuclei were stained with DAPI (Roche). Images were captured with a Nikon Eclipse 90i microscope.

Bioinformatic analyses. We selected a subset of 271 transcripts that included: (a) transcripts mutated in human ciliopathies; (b) transcripts that, when mutated in animal models, give rise to ciliary dysfunction; (c) a group of transcripts found in at least three of the available datasets of ciliary proteins (50); and (d) a subset of transcripts recently shown to be modulators of ciliogenesis and cilium length (51). The publicly available online tool (http://netview.tigem.it) was used to analyze the regulatory interactions among genes from genome-scale measurements of gene expression profiles (microarrays) (32).

ES cell-derived in vitro neural differentiation. The Ofd1-deficient E14Tg2A.4 KOES line was obtained from BayGenomics. Both WT and Ofd1 KOESs were maintained in an undifferentiated state by culture on a monolayer of mitomycin $\mathrm{C}$-inactivated fibroblasts in the presence of leukemia-inhibiting factor (LIF). To induce neural differentiation, we followed previously described protocols (33). Briefly, 48 hours after ES cells were seeded on gelatin-coated plates, they were dissociated and plated on gelatin-coated plates at $1,000 \mathrm{cells} / \mathrm{cm}^{2}$ on day $0(\mathrm{~T} 0)$. The culture medium for neuronal differentiation (serum-free KnockOut Serum Replacement-supplemented medium; Invitrogen) contained knockout DMEM supplemented with 15\% KSR (Invitrogen), 2 mM L-glutamine, $100 \mathrm{U} / \mathrm{ml}$ penicillin-streptomycin, and $0.1 \mathrm{mM} \beta$-mercaptoethanol and was replaced daily during the differentiation process.

Cell culture, DNA transfection, and drug treatment. HEK-293 or HEK293-FT cells and human dermal fibroblasts were grown in DMEM (Invitrogen) containing 10\% FBS (Invitrogen) and $2 \mathrm{mM} \mathrm{L-glutamine}$ (Invitrogen), hTERT-RPE1 cells in DMEM and Ham's F-12 Nutrient 1:1 mixture (DMEM/F-12; Invitrogen) with $10 \% \mathrm{FBS}$ and $2 \mathrm{mM} \mathrm{L-glutamine.}$ FuGene6 Transfection Reagent (Roche) was used for transfection of expression constructs (including the Notch1-NICD expression construct, a gift from Nicholas Gaiano, Johns Hopkins University, Baltimore, Maryland, USA), shRNA-expressing plasmids, and luciferase reporter plasmids; then cells were cultured for 72 hours. Drug treatment was carried out at a final concentration of $10 \mu \mathrm{M}$ SFN (Sigma-Aldrich) for 6 to 24 hours, $30 \mu \mathrm{m} \mathrm{N}$-carbobenzoxyl-L-leucinyl-L-leucinyl-L-norleucinal (MG132; Calbiochem) for 5 hours, $20 \mu \mathrm{M}$ lactacystin (EMD Bioscience) for 5 hours, and $50 \mathrm{ng} / \mathrm{ml}$ TNF- $\alpha$ (Sigma-Aldrich) for 12 hours.

$I P$. For IP, approximately $1 \mathrm{mg}$ of whole-cell, embryo, or tissue lysate was incubated with anti-OFD1, anti-Flag, or anti-GFP at $4^{\circ} \mathrm{C}$ overnight, followed by incubation with protein G-coupled agarose beads (Santa Cruz Biotechnology Inc.) at $4^{\circ} \mathrm{C}$ for 1 hour, or directly with anti-Flag M2 beads (A2220 Sigma-Aldrich). The beads were collected and washed with IP buffer (10\% glycerol, $50 \mathrm{mM}$ Tris- $\mathrm{HCl}$ [pH 7.5], $2.5 \mathrm{mM} \mathrm{MgCl} 2,1 \% \mathrm{NP} 40$, and $200 \mathrm{mM} \mathrm{NaCl}$ ). Proteins conjugated with the beads were then denatured and separated from the beads by boiling at $95^{\circ} \mathrm{C}$ to $100^{\circ} \mathrm{C}$ for 5 minutes before processing for immunoblotting.

Affinity purification of proteasome complex. The $26 \mathrm{~S}$ proteasome complex was purified following a previously described protocol (52) with modifications. Briefly, HEK-293-FT cells expressing stable HTBH-tagged hRPN11 (a gift from Lan Huang, University of California, Irvine, California, USA) were transfected with either $p$ Super control plasmid or pSuperBBS4 to knock down BBS4 expression. Seventy-two hours after transfection, cells were lysed in buffer A $(100 \mathrm{mM} \mathrm{NaCl}, 50 \mathrm{mM}$ Tris- $\mathrm{HCl}$ [pH 7.5], 10\% glycerol, $2 \mathrm{mM} \mathrm{ATP,} 1 \mathrm{mM} \mathrm{DTT}$, and $5 \mathrm{mM} \mathrm{MgCl}_{2}$ ) with $1 \times$ proteasome inhibitor (Roche). Lysates were centrifuged at $4{ }^{\circ} \mathrm{C}$ for 15 minutes to remove cell debris. To purify proteasomes, an aliquot of the supernatant was incubated with streptavidin beads at $4{ }^{\circ} \mathrm{C}$ overnight to precipitate HTBH-RPN11. The beads were then washed with buffer A three times, followed by one washing with TEB buffer $(50 \mathrm{mM}$ Tris-HCl, $\mathrm{pH} 7.5$ and $10 \%$ glycerol). Finally, the beads were incubated in TEB buffer containing $1 \% \mathrm{TEV}$ protease at $30^{\circ} \mathrm{C}$ for 1 hour, before SDS-PAGE and immunoblotting with anti-RPN10 (ab20239; Abcam).

Immunocytochemistry. HEK-293 cells cultured on coverslips were fixed in methanol, blocked in normal goat serum (1:10 in PBS containing 5\% BSA), and then probed with anti-RPN10 and anti- $\gamma$-tubulin, followed by secondary antibodies Alexa Fluor 488 IgG and Alexa Fluor 568 IgG. Finally, nuclei were visualized with Hoechst 33258 (Sigma-Aldrich). Images were captured with a Zeiss LSM 710 confocal microscope and analyzed with ImageJ $1.44 \mathrm{p}$ software.

Sucrose gradient sedimentation. HEK-293-FT cells were transfected and treated with nocodazole $(10 \mu \mathrm{g} / \mathrm{ml})$ and cytochalasin B $(5 \mu \mathrm{g} / \mathrm{ml})$ for 1 hour at 72 hours after transfection. To collect cytoplasmic lysates, cells were harvested in lysis buffer (1 mM HEPES [pH 7.3], 0.5\% NP-40, $0.5 \mathrm{mM}$ $\mathrm{MgCl}_{2}, 0.1 \% \beta$-ME, and $1 \times$ protease inhibitor), followed by centrifugation at 2,500 $\mathrm{g}$ for 10 minutes. After $10 \mathrm{mM}$ HEPES and $5 \mathrm{U} / \mathrm{ml}$ DNase treatment for 30 minutes on ice, $1 \mathrm{ml}$ of cytoplasmic lysates was layered on a discontinued sucrose gradient $(70 \%, 50 \%$, and $40 \%$ sucrose in the buffer containing $10 \mathrm{mM}$ PIPES [pH 7.2], 0.1\% NP-40, and 0.1\% $\beta$-ME) and centrifuged for 1 hour at $195,000 \mathrm{~g} ; 2 \%$ of lysates were kept before ultracentrifugation and served as an input. After ultracentrifugation, 13 fractions were collected and analyzed by immunoblotting.

Microinjection of morpholinos, $m R N A$, and sulforaphane. Morpholinos against $b b s 1$ ( $5^{\prime}$-CACACGTCCATCACTAACCAATAGC-3'), bbs 4 (5'-CCGTTCTCATAGCGTCGTCCGCCAT-3'), and ofd1 (5'-ATCTTCTCTACTGCAACACACATAC-3') were purchased from Gene Tools, LLC. Human RPN10, RPN13, and RPT6 mRNA were in vitro transcribed with an mMESSAGE mMACHINE SP6 Kit (Ambion). SFN was dissolved in DMSO (Sigma-Aldrich) at a stock concentration of $1 \mathrm{M}$ and further diluted in water to $10 \mathrm{mM}$. The morpholino and mRNA or SFN were mixed, and a volume of $0.5 \mathrm{nl}$ was microinjected.

Whole-mount RNA in situ hybridization. Zebrafish embryos were fixed overnight in $4 \%$ PFA at $4{ }^{\circ} \mathrm{C}$. Residual pigment was removed by bleaching with $3 \% \mathrm{H}_{2} \mathrm{O}_{2} / 0.5 \% \mathrm{KOH}$. Whole-mount RNA in situ hybridization was performed with a digoxigenin-labeled anti-her4 RNA probe (a gift from Tohru Ishitani, Kyush University, Fukuoka, Kyushu, Japan) synthesized by in vitro transcription (Roche), followed by immunological detection with Anti-Digoxigenin-AP, Fab Fragments (Roche) and nitro blue tetrazolium/5-bromo-4-chloro-3-indolyl-phosphate staining (Roche). 
Luciferase reporter system assays. HEK-293-FT cells were seeded in 24-well plates at a density of $10^{4}$ cells/well. After 24 hours, cells were transfected with expression constructs, short-hairpin plasmids, and a $3 \mathrm{X}-\mathrm{\kappa B}-\mathrm{L}$ reporter (a gift from Tom Gilmore, Boston University, Boston, Massachusetts, USA) for NF-кB signaling. A pRL-SV40 plasmid expressing Renilla luciferase was used as an internal control. Seventy-two hours after transfection, cells were lysed with Passive Lysis Buffer (Promega). The luciferase activity of lysates was measured with the Dual Luciferase Reporter Assay System (Promega) on a FLUOstar Omega microplate reader (BMG LABTECH) and analyzed with MARS Data Analysis Software (BMG LABTECH).

Generation of Ofd $1^{f / y}$ CAG-Cre-ER $R^{T M}$ mice by tamoxifen injections. Ofd $1^{f /+}$ females were crossed with $C A G-C r e-E R^{T M}$ mice, a general deletor Cre line in which Cre-ER is ubiquitously expressed after tamoxifen injection. We treated pregnant mothers with a single i.p. injection of $100 \mu \mathrm{g}$ tamoxifen/g of weight at E18.5. Tamoxifen (Sigma-Aldrich) was diluted in 10\% ethanol and $90 \%$ sesame oil at a final concentration of $10 \mathrm{mg} / \mathrm{ml}$. Quantification of $O f d 1$ inactivation was assessed by quantitative RT-PCR (qRT-PCR) (data not shown).

Real-time qRT-PCR analyses. Total RNA was isolated with TRIzol (Invitrogen). The cDNA was synthesized from $5 \mu \mathrm{g}$ of total RNA using SuperScript III (Invitrogen). qRT-PCR was performed with Power SYBR Green PCR Master Mix (Applied Biosystems) on a 7900HT Fast Real-Time PCR System (Applied Biosystems). The primers Nfkbib forward (5'-TTGGCTACGTCACTGAGGATG-3') and Nfkbib reverse (5'-GCTCATGCTGATGAATCACAGC-3') were used to test mRNA levels of Nfkbib, while the primers ofd1 forward (5'-TGGCAGACCACTTACAAAGATG-3') and of $f 1$ reverse (AGACTGGATGAGGGGTTAATC- $3^{\prime}$ ) were used to examine the conditional knockout efficiency, and the primers gapdh forward ( $5^{\prime}$-TCTTCTGGGTGGCAGTGAT-3') and gapdh reverse (5'-TGCACCACCAACTGCTTAGC-3') were used as internal controls. Real-time data were collected and analyzed with the Sequence Detection System software package, version 2.3 (Applied Biosystems).

Statistics. A one-tailed Student's $t$ test was performed to compare the means of two populations. In the bar graphs, data represent the mean \pm SEM of multiple repeats $(n \geq 3)$. A $\chi^{2}$ test was performed to compare two populations with several subgroups of different proportions. Statistical significance of differences between samples are indicated by ${ }^{*} P<0.05$, ${ }^{*} P<0.01$, and ** $P<0.001$. A $P$ value less than 0.05 was considered significant.
Study approval. Zebrafish and mouse studies were approved by the IACUC of Duke University (protocol A229-12-08 and A251-12-09) and by the IACUC of Cardarelli Hospital (Naples, Italy), to which the Telethon Institute of Genetics and Medicine (TIGEM) refers, and were authorized by the Italian Ministry of Health. The Cardarelli Hospital Ethics Committee (Naples, Italy) approved this study.

\section{Acknowledgments}

We thank Gopuraja Dharmalingam and the TIGEM Bioinformatic Core for the bioinformatic analysis, Nicholas Gaiano (Johns Hopkins University) for the Notch1-NICD expression constructs, Tom Gilmore (Boston University) for the 3X-кB-L reporter, Lan Huang (University of California, Irvine) for the HEK-293 cells stably expressing HTBH-hRPN11, Tohru Ishitani (Kyush University) for the her4 probe plasmid, and Jeremy Nathans (Johns Hopkins University) for the anti-s-opsin antibody. This work was supported by grants from the NIH (NRSA fellowship F32DK094578, to I.-C. Tsai; R37GM04360, to D. Finley; K01DK092402, to N. A. Zaghloul; and R01HD04260, R01DK072301, and R01DK075972, to N. Katsanis), the Macular Vision Research Foundation and the Foundation for Fighting Blindness (to N. Katsanis), Telethon (TGM11CB3, to B. Franco), and the 7th Framework Large Integrated Project Syscilia (241955, to N. Katsanis and B. Franco). E.C. Oh is a NARSAD Young Investigator Award recipient. N. Katsanis is a Distinguished George W. Brumley Professor.

Received for publication July 1, 2013, and accepted in revised form February 6, 2014.

Address correspondence to: Brunella Franco, Telethon Institute of Genetics and Medicine, Via Pietro Castellino 111, 80131 Naples, Italy. Phone: 39.081.6132207; Fax: 39.081.5790919; E-mail: franco@tigem.it. Or to: Nicholas Katsanis, 466A Nanaline Duke Building, Box 3709, Duke University Medical Center, Durham, North Carolina 27710, USA. Phone: 919.613.4694; Fax: 919.684.1627; E-mail: katsanis@cellbio.duke.edu.
1. Hildebrandt F, Benzing T, Katsanis N. Ciliopathies. NEngl J Med. 2011;364(16):1533-1543.

2. Eggenschwiler JT, Anderson KV. Cilia and developmental signaling. Annu Rev Cell Dev Biol. 2007; 23:345-373.

3. Lancaster MA, Gleeson JG. Cystic kidney disease: the role of Wnt signaling. Trends Mol Med. 2010;16(8):349-360.

4. Ocbina PJ, Tuson M, Anderson KV. Primary cilia are not required for normal canonical Wnt signaling in the mouse embryo. PLoS One. 2009;4(8):e6839.

5. Gerdes JM, et al. Disruption of the basal body compromises proteasomal function and perturbs intracellular Wnt response. Nat Genet. 2007; 39(11):1350-1360.

6. Lancaster MA, Schroth J, Gleeson JG. Subcellular spatial regulation of canonical Wnt signalling at the primary cilium. Nat Cell Biol. 2011;13(6):700-707.

7. Simons $M$, et al. Inversin, the gene product mutated in nephronophthisis type II, functions as a molecular switch between Wnt signaling pathways. Nat Genet. 2005;37(5):537-543.

8. Corbit KC, et al. Kif3a constrains $\beta$-catenin-dependent Wnt signalling through dual ciliary and non-ciliary mechanisms. Nat Cell Biol. 2008; 10(1):70-76.

9. Oh EC, Katsanis N. Context-dependent regulation of Wnt signaling through the primary cilium. J Am Soc Nephrol. 2013;24(1):10-18.

10. Corbit KC, Aanstad P, Singla V, Norman AR,
Stainier DY, Reiter JF. Vertebrate Smoothened functions at the primary cilium. Nature. 2005; 437(7061):1018-1021.

11. Haycraft CJ, Banizs B, Aydin-Son Y, Zhang Q, Michaud EJ, Yoder BK. Gli2 and Gli3 localize to cilia and require the intraflagellar transport protein polaris for processing and function. PLoS Genet. 2005;1(4):e53.

12. Schneider L, et al. PDGFRalphaalpha signaling is regulated through the primary cilium in fibroblasts. Curr Biol. 2005;15(20):1861-1866.

13. Ezratty EJ, Stokes N, Chai S, Shah AS, Williams SE, Fuchs E. A role for the primary cilium in Notch signaling and epidermal differentiation during skin development. Cell. 2011;145(7):1129-1141.

14. Cano DA, Murcia NS, Pazour GJ, Hebrok M. Orpk mouse model of polycystic kidney disease reveals essential role of primary cilia in pancreatic tissue organization. Development. 2004;131(14):3457-3467.

15. Dafinger C, et al. Mutations in KIF7 link Joubert syndrome with Sonic Hedgehog signaling and microtubule dynamics. J Clin Invest. 2011;121(7):2662-2667.

16. Putoux A, et al. KIF7 mutations cause fetal hydrolethalus and acrocallosal syndromes. Nat Genet. 2011; 43(6):601-606.

17. Kim SK, et al. Planar cell polarity acts through septins to control collective cell movement and ciliogenesis. Science. 2010;329(5997):1337-1340.

18. Wigley WC, et al. Dynamic association of proteasomal machinery with the centrosome. J Cell Biol.
1999;145(3):481-490.

19. Fabunmi RP, Wigley WC, Thomas PJ, DeMartino GN. Activity and regulation of the centrosome-associated proteasome. J Biol Chem. 2000; 275(1):409-413.

20. Li J, et al. USP33 regulates centrosome biogenesis via deubiquitination of the centriolar protein CP110. Nature. 2013;495(7440):255-259.

21. Mahuzier A, et al. Dishevelled stabilization by the ciliopathy protein Rpgrip1l is essential for planar cell polarity. J Cell Biol. 2012;198(5):927-940.

22. Itoh K, Jenny A, Mlodzik M, Sokol SY. Centrosomal localization of Diversin and its relevance to Wnt signaling. J Cell Sci. 2009; 122(pt 20):3791-3798.

23. Chitnis $A B$. The role of Notch in lateral inhibition and cell fate specification. Mol Cell Neurosci. 1995;6(6):311-321.

24. Lai EC, Deblandre GA, Kintner C, Rubin GM. Drosophila neuralized is a ubiquitin ligase that promotes the internalization and degradation of delta. Dev Cell. 2001;1(6):783-794.

25. Kopan R. All good things must come to an end: how is Notch signaling turned off? Sci STKE. 1999;1999(9):PE1.

26. Aza-Blanc P, Ramirez-Weber FA, Laget MP, Schwartz C, Kornberg TB. Proteolysis that is inhibited by hedgehog targets Cubitus interruptus protein to the nucleus and converts it to a repressor. Cell. 1997;89(7):1043-1053. 
27. Pan Y, Bai CB, Joyner AL, Wang B. Sonic hedgehog signaling regulates Gli2 transcriptional activity by suppressing its processing and degradation. Mol Cell Biol. 2006;26(9):3365-3377.

28. Chen $\mathrm{MH}$, et al. Cilium-independent regulation of Gli protein function by Sufu in Hedgehog signaling is evolutionarily conserved. Genes Dev. 2009;23(16):1910-1928.

29. Ross AJ, et al. Disruption of Bardet-Biedl syndrome ciliary proteins perturbs planar cell polarity in vertebrates. Nat Genet. 2005;37(10):1135-1140.

30. Ferrante MI, et al. Convergent extension movements and ciliary function are mediated by ofd 1 , a zebrafish orthologue of the human oral-facial-digital type 1 syndrome gene. Hum Mol Genet. 2009; 18(2):289-303.

31. Lindsten K, Menendez-Benito V, Masucci MG, Dantuma NP. A transgenic mouse model of the ubiquitin/proteasome system. Nat Biotechnol. 2003; 21(8):897-902.

32. Belcastro V, et al. Transcriptional gene network inference from a massive dataset elucidates transcriptome organization gene function. Nucleic Acids Res. 2011;39(20):8677-8688

33. Fico A, Manganelli G, Simeone M, Guido S, Minchiotti G, Filosa S. High-throughput screening-compatible single-step protocol to differentiate embryonic stem cells in neurons. Stem Cells Dev. 2008;17(3):573-584.

34. Singla V, Romaguera-Ros M, Garcia-Verdugo JM, Reiter JF. Ofd1, a human disease gene, regulates the length and distal structure of centrioles. Dev Cell. 2010;18(3):410-424.
35. Pan Y, Wang B. A novel protein-processing domain in Gli2 and Gli3 differentially blocks complete protein degradation by the proteasome. J Biol Chem. 2007;282(15):10846-10852.

36. Kwak MK, Cho JM, Huang B, Shin S, Kensler TW. Role of increased expression of the proteasome in the protective effects of sulforaphane against hydrogen peroxide-mediated cytotoxicity in murine neuroblastoma cells. Free Radic Biol Med. 2007;43(5):809-817.

37. Lee BH, et al. Enhancement of proteasome activity by a small-molecule inhibitor of USP14. Nature. 2010;467(7312):179-184.

38. Zaghloul NA, et al. Functional analyses of variants reveal a significant role for dominant negative and common alleles in oligogenic BardetBiedl syndrome. Proc Natl Acad Sci U S A. 2010; 107(23):10602-10607.

39. Jiang YJ, Aerne BL, Smithers L, Haddon C, Ish-Horowicz D, Lewis J. Notch signalling and the synchronization of the somite segmentation clock. Nature. 2000;408(6811):475-479.

40. Takke C, Dornseifer P, v Weizsacker E, Campos-Ortega JA. her4, a zebrafish homologue of the Drosophila neurogenic gene $\mathrm{E}(\mathrm{spl})$, is a target of NOTCH signalling. Development. 1999; 126(9):1811-1821.

41. Shih VF, Tsui R, Caldwell A, Hoffmann A. A single NFKB system for both canonical and non-canonical signaling. Cell Res. 2011;21(1):86-102.

42. Hamazaki J, Sasaki K, Kawahara H, Hisanaga S, Tanaka K, Murata S. Rpn10-mediated degradation of ubiquitinated proteins is essential for mouse development. Mol Cell Biol. 2007;27(19):6629-6638.

43. Hanna J, Meides A, Zhang DP, Finley D. A ubiquitin stress response induces altered proteasome composition. Cell. 2007;129(4):747-759.

44. Jin $\mathrm{H}$, et al. The conserved Bardet-Biedl syndrome proteins assemble a coat that traffics membrane proteins to cilia. Cell. 2010;141(7):1208-1219.

45. Oeffner F, Moch C, Neundorf A, Hofmann J, Koch M, Grzeschik KH. Novel interaction partners of Bardet-Biedl syndrome proteins. Cell Motil Cytoskeleton. 2008;65(2):143-155.

46. Garcia-Gonzalo FR, et al. A transition zone complex regulates mammalian ciliogenesis and ciliary membrane composition. Nat Genet. 2011;43(8):776-784.

47. Huang P, Schier AF. Dampened Hedgehog signaling but normal Wnt signaling in zebrafish without cilia. Development. 2009;136(18):3089-3098.

48. Egner PA, et al. Bioavailability of Sulforaphane from two broccoli sprout beverages: results of a shortterm, cross-over clinical trial in Qidong, China. Cancer Prev Res (Phila). 2011;4(3):384-395.

49. Shapiro TA, et al. Safety, tolerance, and metabolism of broccoli sprout glucosinolates and isothiocyanates: a clinical phase I study. Nutr Cancer. 2006;55(1):53-62.

50. Inglis PN, Boroevich KA, Leroux MR. Piecing together a ciliome. Trends Genet. 2006;22(9):491-500.

51. Kim J, et al. Functional genomic screen for modulators of ciliogenesis and cilium length. Nature. 2010;464(7291):1048-1051.

52. Wang X, Chen CF, Baker PR, Chen PL, Kaiser P, Huang L. Mass spectrometric characterization of the affinity-purified human $26 \mathrm{~S}$ proteasome complex. Biochemistry. 2007;46(11):3553-3565. 